\title{
HEMODYNAMIC EFFECT OF PROPHYLACTIC SURFACTANT REPLACEMENT THERAPY WITH RAPID EXTUBATION TO NASAL CPAP
}

\author{
K. de Waal ${ }^{1,2}$, A. Lakkundi ${ }^{1}$, I. Wright ${ }^{1,2}$ \\ ${ }^{1}$ Neonatology, John Hunter Hospital, ${ }^{2}$ University of Newcastle, Newcastle, NSW, Australia
}

Background: The practice of intubation, surfactant administration, and rapid extubation to CPAP (INSURE) has been gaining popularity based on published observations and reduces the need for mechanical ventilation during transition. The aim of this study is to explore the effect of INSURE on transitional hemodynamics.

Methods: In our unit, inborn infants $<29$ week gestation without significant perinatal asphyxia or major congenital abnormalities undergoes INSURE within 30 minutes of birth using $200 \mathrm{mg} / \mathrm{kg}$ Curosurf. Blood pressure (BP) and central blood flow parameters (RVO, LVO, SVC flow, ductal size) were measured at 6, 24 and 72 hours of age and information on morbidity was collected.

Results: Forty seven infants with a median (range) weight of 940 (450-1270) grams have been studied thus far. There were $9(19 \%)$ patients needing mechanical ventilation within 72 hours of life (INSURE failure). Five (11\%) patients had a BP < gestational age and $5(11 \%)$ patients had low blood flow (defined as SVC flow $<45$, LVO or RVO $<150 \mathrm{ml} / \mathrm{kg} / \mathrm{min}$ ). LVO and RVO, not SVC flow, showed a pattern of slow increase over the first 72 hours of life ( $F$ 5.6, $p=0.030, F 11.2, p<0.001$ and $F 1.1, p=0.357$ respectively). Mortality in the first week ( 5 patients) or $\mathrm{P} / \mathrm{IVH}>$ grade 1 (4 patients) was more common in infants with low blood flow.

Conclusion: This data suggests a lower prevalence of low blood pressure and low blood flow with INSURE as compared to reported cohorts where mechanical ventilation was preferred during transition. 